\title{
Prospective Multicenter Surveillance Study of Surgical Site Infection after Intracranial Procedures in Korea : A Preliminary Study
}

\author{
Tae Seok Jeong, M.D., Gi Taek Yee, M.D., Ph.D. \\ Department of Neurosurgery, Gil Medical Center, Gachon University College of Medicine, Incheon, Korea
}

Objective : This study aimed to investigate the rates, types, and risk factors of surgical site infection (SSI) following intracranial neurosurgical procedures evaluated by a Korean SSI surveillance system.

Methods : This was a prospective observational study of patients who underwent neurosurgical procedures at 29 hospitals in South Korea from January 2017 to June 2017. The procedures included craniectomy, craniotomy, cranioplasty, burr hole, and ventriculoperitoneal shunt. Univariate and multivariate logistic regression analyses were performed.

Results : Of the 1576 cases included, 30 showed infection, for an overall SSI rate of 1.9\%. Organ/space infection was the most common, found in 21 out of the 30 cases (70\%). Staphylococcus aureus was the most common (41\%) of all bacteria, and Serratia marcescens (12\%) was the most common among gram-negative bacteria. In univariate analyses, the $p$-values for age, preoperative hospital stay duration, and over T-hour were $<0.2$. In a multivariate analysis of these variables, only preoperative hospital stay was significantly associated with the incidence of SSI $(p<0.001)$, whereas age and over T-hour showed a tendency to increase the risk of SSI ( $p=0.09$ and 0.06$)$.

Conclusion : Surveillance systems play important roles in the accurate analysis of SSI. The incidence of SSI after neurosurgical procedures assessed by a national surveillance system was $1.9 \%$. Future studies will provide clinically useful results for SSI when data are accumulated.

Key Words : Surgical site infection · Neurosurgery · Risk factors · Korea · Incidence.

\section{INTRODUCTION}

Surgical site infection (SSI) is the third most frequently reported nosocomial infection, accounting for $14-16 \%$ of all infections among hospitalized patients and 38\% among surgical patients ${ }^{9,19)}$. SSI is associated with substantial morbidity and mortality, prolonged hospital stay, and increased expenses ${ }^{24}$.
Although the SSI rate after neurosurgery is generally known to be lower than that after other surgery, SSI can cause catastrophic consequences such as altered consciousness and the need for reoperation. Reoperation is a burden to both the patient and physician. Reported rates of SSI following intracranial neurosurgical procedures have been variable, ranging from $<1 \%$ to $>8 \%$ in a published series ${ }^{20}$. After neurosurgical

- Received : January 28, 2018 •Revised : March 13, 2018 •Accepted : April 24, 2018

- Address for reprints : Gi Taek Yee, M.D., Ph.D.

Department of Neurosurgery, Gil Medical Center, Gachon University College of Medicine, 21 Namdong-daero 774beon-gil, Namdong-gu, Incheon 21565, Korea Tel : +82-32-460-3304, Fax : +82-32-460-3899, E-mail : gtyee@gilhospital.com

This is an Open Access article distributed under the terms of the Creative Commons Attribution Non-Commercial License (http://creativecommons.org/licenses/by-nc/4.0) which permits unrestricted non-commercial use, distribution, and reproduction in any medium, provided the original work is properly cited. 
procedures, SSI most commonly presents as meningitis, epidural abscess, subdural empyema, or brain abscess ${ }^{14)}$.

Many efforts have been made to prevent SSI, one of which is the use of infection surveillance systems. The Korean National Healthcare-Associated Infection Surveillance System (KONIS) is operated by the Korean Centers for Disease Control and Prevention (CDC). It monitors the current status of SSI in Korea as a whole and helps develop means to reduce SSI rate. Hospitals of all sizes are enrolled in the KONIS system. This study aimed to investigate the rates, types, and risk factors for SSI following intracranial neurosurgical procedures.

\section{MATERIALS AND METHODS}

This was a prospective observational study of patients who underwent neurosurgical procedures at a total of 29 hospitals in South Korea from January 2017 to June 2017. The neurosurgical procedures included craniectomy, craniotomy, cranioplasty, burr hole, and ventriculoperitoneal (VP) shunt. Patients who survived at least 7 days after surgery were included in the study. Prophylactic antibiotics were given to all patients in accordance with the Ministry of Health guidelines. The first-generation cephalosporin was administered within 1 hour before skin incision, and the duration of administration did not exceed 48 hours after surgery. Patients who under 18 years of age, be transferred, with a fever greater than 38 degrees within 24 hours before surgery, with American Society of Anesthesiologists (ASA) class 4 or more, underwent more than two operations during the hospital stay and underwent emergency operations were excluded from prophylactic antibiotics guidelines. For surveillance, surgical procedures were classified according to the KONIS-SSI code, referencing the 2017 electronic data interchange (EDI) insurance code. The KONIS-SSI codes for the surgical procedures in this study were craniotomy (CRAN) and ventricular shunt (VSHN). Table 1 shows the Health Insurance EDI codes for CRAN and VSHN. Patients were monitored for 90 days after surgery; those discharged or transferred were monitored at outpatient clinics. Patients who underwent reoperation, passed away within 48 hours after surgery, or did not have the incision site closed were excluded.

Data were collected by the surgeon performing the surgery and nurse practitioners who had completed the KONIS-SSI steering committee course at least once a year. Data included were age, sex, duration of preoperative hospital stay, elective or emergency surgery, endoscopic procedure, combined surgery, reoperation, surgery by trauma, operation time, wound classification (clean, clean-contaminated, contaminated, and dirty) ${ }^{22)}$, ASA classification ${ }^{1)}$, National Nosocomial Infection Surveillance (NNIS)-derived risk index ${ }^{11)}$, infection, and infecting microorganisms. These values were recorded on standardized worksheets specially designed according to the guidelines in the KONIS-SSI manual.

\section{SSI criteria and wound classification}

The criteria used to define SSI were established by the CDC in $2017^{4,5)}$. In accordance with these criteria, SSI was defined as any infection occurring within 1 month of the operation when no prosthetic material was left in the wound, or within 1 year when prosthetic material remained within the operation site. However, since the monitoring period was 90 days, SSI was evaluated as an infection within 90 days even if the prosthetic material was inserted. Metal plates and screws used to fix the

Table 1. KONIS-SSI and EDI codes for neurosurgical procedures included in the study

\begin{tabular}{|c|c|c|c|}
\hline KONIS-SSI code & Type of surgery & Definition and surveillance scope & 2017 Health Insurance EDI standard code \\
\hline CRAN & Craniotomy & $\begin{array}{l}\text { The skull is opened to expose the brain or the } \\
\text { meninges, except for a simple puncture or } \\
\text { a burr hole. }\end{array}$ & $\begin{array}{l}\text { N0331, N0333, N0334, N0335, S0471, S0472, S0475, S0476, } \\
\text { S4621, S4622, S4633, S4634, S4635, S4636, S4637, S4641, } \\
\text { S4642, S4653, S4654, S4655, S4656, S4657, S4658, S4661, } \\
\text { S4662, S4681, S4683, S4733, S4734, S4735, S4736, S4737, } \\
\text { S4760, S4771, S4772, S4780, S4792, S4793, S4794, S4796, } \\
\text { S4797, S4798, S4799, S4801, S4802, S4803 }\end{array}$ \\
\hline VSHN & Ventricular shunt & $\begin{array}{l}\text { A short circuit is inserted from the ventricle to } \\
\text { another site; includes correction or removal } \\
\text { of a shunt. }\end{array}$ & $\$ 4712$ \\
\hline
\end{tabular}

KONIS : Korean National Healthcare-Associated Infection Surveillance System, SSI : surgical site infection, EDI : electronic data interchange, CRAN : craniotomy, VSHN : ventricular shunt 
bone flap were considered prosthetic material. SSIs were classified depending on the degree of infection as follows : superficial incisional, deep incisional, and organ/space. These categories involved skin or subcutaneous tissue at the incision, deep soft tissue (fascia and muscle layers) at the incision, or any part of the anatomy (organs or organ spaces), respectively. Because no clear distinction exists between superficial and deep incisional infection in brain surgery, we included skin or subcutaneous tissue in the definition of superficial incisional infection, and fascia/muscle layers and aponeurosis/skull bone in that of deep incisional infection. Organ/space infection was diagnosed as infection of the central nervous system, such as intracranial infection (brain abscess, subdural or epidural infection, encephalitis), and meningitis or ventriculitis ${ }^{5)}$.

A method suggested by Narotam et al. ${ }^{22)}$ was applied to wound classification in neurosurgical procedures as follows : "dirty" procedures included brain abscess, subdural empyema, and osteitis surgical treatments when sepsis was already present; "contaminated" procedures included mainly trauma patients with open compound cranial fractures or scalp lacerations older than 4 hours; "clean-contaminated" procedures included paranasal sinuses or mastoid entry, repair of cranial base fractures, and aseptic surgical technique breaches; "clean" procedures included most of the scheduled surgeries.

\section{Statistical analysis}

To compare differences in variables between the SSI and non-SSI groups, Fisher's exact test or the Wilcoxon rank sum test were used. Spearman correlation analysis was used to examine the correlation between hospital scale and SSI rate. $p$ values of $<0.05$ were considered significant. Univariate and multivariate logistic regression analyses were conducted with SAS version 9.4 (SAS Institute Inc., Cary, NC, USA). The objective of the univariate analysis was to determine the risk factors linked to SSI, followed by multivariate analysis for variables with $p$-values $<0.2$.

\section{RESULTS}

\section{Population}

A total of 1576 cases (CRAN, 1495; VSHN, 81) fulfilled the inclusion criteria and were enrolled in the study from September 2016 to June 2017. Comparisons between the SSI and non-
SSI groups were carried out with VP shunt cases (VSHN) excluded because of the low number of SSIs (two cases). For the CRAN code, the mean age of the non-SSI group was significantly higher than that of the SSI group (57 vs. 52 years; $p=0.045$ ), and the mean preoperative hospital stay was significantly longer in the SSI group than in the non-SSI group (13 vs. 28 days; $p=0.014)$. No difference was found between the groups in the ASA class (Table 2).

\section{Surgical procedures}

For the variables of endoscopic, emergency, combined, reoperation, and trauma, no significant differences were found between the two groups. In addition, no difference was observed in wound classification. Although the differences in mean operation time and over T-hour (the exact 75th percentile of procedure duration in minutes) were not significant, over T-hour in the SSI group tended to be higher than in the non-SSI group (24.7 vs. $39.3 \%$; $p=0.077$ ) (Table 2 ).

\section{SSI incidence}

We investigated the SSI rate by NNIS risk index category. For CRAN, the SSI rate was higher as the risk index increased from $1.39 \%$ for risk index 0 to $2.59 \%$ for risk index 2 . Risk index 3 was not significantly different because it was only present in two cases. The overall SSI rate for CRAN was 1.9\%. For VSHN, risk index 0 showed an SSI rate of 9.5\%; no SSI occurred with risk index 1 or 2 . No surgical cases had risk index 3. The overall SSI rate for VSHN was $2.5 \%$ (Table 3). In CRAN cases, organ/space infection was the most common, present in 19 out of 28 cases. The 2 VSHN cases were organ/space infection (Table 4). The overall SSI rates for CRAN and VSHN varied depending on hospital size; however, the correlation was not significant ( $p=0.702$, Table 5). Staphylococcus aureus was the most common (41\%) causative organism among all bacteria, and Serratia marcescens (12\%) among gram-negative bacteria (Fig. 1).

\section{SSI risk factors}

Univariate and multivariate analyzes were performed to identify risk factors for SSI. In univariate analyses, the $p$-values for age, preoperative hospital stay duration, and over Thour were $<0.2$. In a multivariate analysis of these three variables, only preoperative hospital stay was significantly associated with SSI incidence $(p<0.001$, Table 6). 
Table 2. General characteristics of non-SSI and SSI groups of CRAN cases

\begin{tabular}{|c|c|c|c|}
\hline & Non-SSI group $(n=1467)$ & SSI group $(n=28)$ & $p$-value \\
\hline Sex (female) & $766(52.2)$ & $14(50.0)$ & 0.816 \\
\hline Age (years) & $57(4-90)$ & $52(25-84)$ & $0.045^{*}$ \\
\hline Endoscopic operation & $78(5.3)$ & 0 & 0.396 \\
\hline Emergency operation & $556(37.9)$ & $13(46.4)$ & 0.357 \\
\hline Trauma & $122(8.3)$ & 0 & 0.162 \\
\hline Combined operation & $31(2.1)$ & 0 & 1.000 \\
\hline Reoperation & $61(4.2)$ & $2(7.1)$ & 0.332 \\
\hline Duration of preoperative hospital stay & $13(0-214)$ & $28(4-300)$ & $0.014^{*}$ \\
\hline \multicolumn{4}{|l|}{ Wound classification } \\
\hline Clean & $1412(96.3)$ & $28(100.0)$ & 1.000 \\
\hline Clean-contaminated & $24(1.6)$ & 0 & \\
\hline Contaminated & $24(1.6)$ & 0 & \\
\hline Dirty & $7(0.5)$ & 0 & \\
\hline \multicolumn{4}{|l|}{ ASA classification } \\
\hline 1 & $178(12.1)$ & $2(7.1)$ & 0.869 \\
\hline 2 & $738(50.3)$ & $15(53.6)$ & \\
\hline 3 & $412(28.1)$ & $10(35.7)$ & \\
\hline 4 & $104(7.1)$ & $1(3.6)$ & \\
\hline 5 & $35(2.4)$ & 0 & \\
\hline Operation time (minutes) & $206(20-1750)$ & $264(80-534)$ & 0.389 \\
\hline Over T-hour ${ }^{\dagger}$ & $362(24.7)$ & $11(39.3)$ & 0.077 \\
\hline
\end{tabular}

Values are presented as median value (interquartile ranges) or number (\%). * Statistically significant differences ( $p$-value $<0.05)$. ${ }^{\dagger}$ The exact 75 th percentile of procedure duration in minutes. SSI : surgical site infection, CRAN : craniotomy, ASA : American Society of Anesthesiologists

Table 3. Pooled mean and key percentiles of the distribution of SSI rates by risk index

\begin{tabular}{|c|c|c|c|c|c|}
\hline KONIS-SSI code & Risk index & Number of participating hospitals & Number of operations & Number of infections & Infection rate (\%) \\
\hline \multirow[t]{5}{*}{ CRAN } & 0 & 23 & 649 & 9 & 1.39 \\
\hline & 1 & 25 & 728 & 16 & 2.20 \\
\hline & 2 & 20 & 116 & 3 & 2.59 \\
\hline & 3 & 2 & 2 & 0 & 0.00 \\
\hline & Total & 25 & 1495 & 28 & 1.87 \\
\hline \multirow[t]{5}{*}{ VSHN } & 0 & 7 & 21 & 2 & 9.52 \\
\hline & 1 & 7 & 46 & 0 & 0.00 \\
\hline & 2 & 5 & 14 & 0 & 0.00 \\
\hline & 3 & 0 & 0 & 0 & 0.00 \\
\hline & Total & 8 & 81 & 2 & 2.47 \\
\hline
\end{tabular}

SSI : surgical site infection, KONIS : Korean National Healthcare-associated Infections Surveillance System, CRAN : craniotomy, VSHN : ventricular shunt

\section{DISCUSSION}

The importance of infection surveillance was acknowledged more than 40 years ago by the CDC and the Joint Commission on Accreditation of Hospitals, and has proven effective in preventing SSIs ${ }^{6,8)}$. The Study on the Efficacy of Nosocomial 
Table 4. Type of SSIs according to operative procedure

\begin{tabular}{lcccccc}
\hline $\begin{array}{c}\text { KONIS-SSI } \\
\text { code }\end{array}$ & Type of surgery & $\begin{array}{c}\text { Number of } \\
\text { operations }\end{array}$ & $\begin{array}{c}\text { Number of } \\
\text { infections }\end{array}$ & $\begin{array}{c}\text { Superficial incisional } \\
\text { infections }\end{array}$ & $\begin{array}{c}\text { Deep incisional } \\
\text { infections }\end{array}$ & $\begin{array}{c}\text { Organ/space } \\
\text { infections }\end{array}$ \\
\hline CRAN & Craniotomy & 1495 & 28 & 5 & 4 & 19 \\
VSHN & Ventricular shunt & 81 & 2 & 0 & 5 & 0 \\
\hline Total & & 1576 & 30 & 4 & 21 \\
\hline
\end{tabular}

SSI : surgical site infection, KONIS : Korean National Healthcare-associated Infections Surveillance System, CRAN : craniotomy, VSHN : ventricular shunt

Table 5. SSI rate by hospital size

\begin{tabular}{lccc|}
\hline $\begin{array}{c}\text { Number of } \\
\text { beds }\end{array}$ & $\begin{array}{c}\text { Number of } \\
\text { operations }\end{array}$ & $\begin{array}{c}\text { Number of } \\
\text { infections }\end{array}$ & $\begin{array}{c}\text { Infection rate } \\
\text { (\%) }\end{array}$ \\
\hline$\leq 299$ & 36 & 0 & 0.00 \\
\hline $300-499$ & 85 & 4 & 4.71 \\
\hline $500-749$ & 287 & 3 & 1.05 \\
\hline $750-899$ & 361 & 7 & 1.94 \\
\hline $900-999$ & 142 & 5 & 3.52 \\
\hline $1000-1499$ & 232 & 2 & 0.86 \\
\hline 1500 & 433 & 9 & 2.08 \\
\hline Total & 1576 & 30 & 1.90 \\
\hline SS1: & & & \\
\hline
\end{tabular}

SSI : surgical site infection

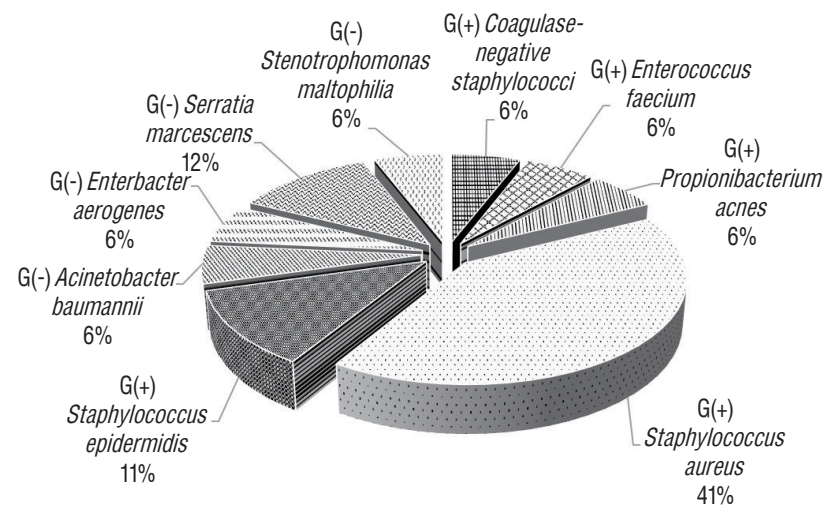

Fig. 1. Microorganisms identified in surgical site infection.

Table 6. Univariate and multivariate analysis of factors influencing SSI in craniotomy (CRAN)

\begin{tabular}{|c|c|c|c|c|c|c|}
\hline & \multicolumn{3}{|c|}{ Univariate } & \multicolumn{3}{|c|}{ Multivariate } \\
\hline & OR & $95 \% \mathrm{Cl}$ & $p$-value & Adjusted OR & $95 \% \mathrm{Cl}$ & $p$-value \\
\hline Sex (female vs. male) & 0.92 & $0.43,1.93$ & 0.816 & & & \\
\hline Age & 0.98 & $0.96,1.01$ & $0.167^{*}$ & 0.98 & $0.95,1.00$ & 0.090 \\
\hline Endoscopic OP (yes vs. no) & $<0.001$ & $<0.001,>999.999$ & 0.971 & & & \\
\hline Emergency OP (yes vs. no) & 1.42 & $0.67,3.01$ & 0.359 & & & \\
\hline Trauma (yes vs. no) & $<0.001$ & $<0.001,>999.999$ & 0.964 & & & \\
\hline Combined OP (yes vs. no) & $<0.001$ & $<0.001,>999.999$ & 0.982 & & & \\
\hline Reoperation (yes vs. no) & 1.77 & $0.41,7.64$ & 0.442 & & & \\
\hline Duration of preoperative hospital stay & 1.02 & $1.02,1.03$ & $<0.001^{*}$ & 1.02 & $1.01,1.03$ & $<0.001^{\dagger}$ \\
\hline $\begin{array}{l}\text { Wound classification (contaminated and dirty vs. } \\
\text { clean and clean-contaminated) }\end{array}$ & $<0.001$ & $<0.001,>999.999$ & 0.982 & & & \\
\hline ASA classification (ASA 1, 2 vs. ASA 3, 4, 5) & 1.08 & $0.50,2.31$ & 0.852 & & & \\
\hline Operation time & 1.00 & $1.00,1.00$ & 0.391 & & & \\
\hline Over T-hour ${ }^{\ddagger}$ & 1.98 & $0.92,4.26$ & $0.082^{*}$ & 3.80 & $0.97,14.88$ & 0.055 \\
\hline
\end{tabular}

${ }^{*} p$-value $<0.2$. ${ }^{\dagger}$ Statistically significant differences ( $p$-value $\left.<0.05\right)$. ${ }^{\ddagger}$ The exact 75 th percentile of procedure duration in minutes. SSI : surgical site infection, CRAN : craniotomy, OR : odds ratio, Cl : confidence interval, OP : operation, ASA : American Society of Anesthesiologists

Infection Control, which investigated whether a surveillance program could reduce the infection rate, concluded based on U.S. data that SSIs can be reduced by up to $32 \%$ if the following four conditions are met : 1) surveillance is continuous, 2) management efforts are aggressive, 3) infection control personnel are qualified, and 4) feedback on the infection rate is provided to the surgeon, especially on SSI ${ }^{13}$. Therefore, continuous SSI surveillance and feedback on the surveillance re- 
sults are important for the prevention of SSI.

In Korea, a nationwide surveillance system for SSI was first applied to orthopedic artificial joint surgery in 2006, and neurosurgical procedures including craniotomy and VP shunt have been included in the survey since 2009. However, because the surgeon's participation in the infection surveillance system has not been mandatory, the accuracy of SSI diagnosis and surveillance is evaluated to have been low. Therefore, the 2017 KONIS manual mandated the participation of surgeons. This study is the first in a national survey of SSI in the neurosurgical field using the 2017 KONIS manual. We analyzed the SSI incidence in the neurosurgical field to find possible means to prevent SSI.

In this study, the overall SSI rate for 1576 cases was 2.1\% (CRAN, 1.9\%; VSHN, 2.5\%). The results were similar to those of previously published studies. The incidence of postoperative wound infection documented in the literature range from as low as $1.25 \%$ to as high as $17 \%$ without, and from $0.3 \%$ to $3.0 \%$ with prophylactic antibiotics ${ }^{15,16,20,22,23)}$. In the present study, the SSI incidence with prophylactic antibiotics was within the previously reported range.

Among the total of 30 infection cases, organ/space infections accounted for $70 \%$, followed by superficial (17\%) and deep (13\%) infections. Patir et al. ${ }^{23)}$ reported 5\% wound infection and 6\% meningitis rates among 413 cases. Korinek et al. ${ }^{16)}$ reported a 5.3\% incision infection rate combined with scalp infection and bone flap osteitis, and a 1.52\% meningitis incidence among 331 cases. Buang and Haspani ${ }^{3)}$ reported scalp incision infection in $4.9 \%$, bone flap osteitis in $2.3 \%$, and organ $/$ space infection in $0.5 \%$ of the cases. Although exact comparison among studies is difficult because of different infection classifications and overall infection rates, we found the organ/space infection rate to be higher than that of incisional infection, in contrast to previous studies.

No studies have found any association between SSI and hospital size. However, nosocomial infection rates have been reported to increase with increasing hospital size ${ }^{10,21)}$, possibly because of infection control personnel qualification, facility hygiene and maintenance, and pathogen variety depending on hospital size. However, the present study did not find any correlation between SSI and hospital size. Because the number of infections was low, an accurate analysis may not be possible, requiring further study.

In this study, gram-positive bacteria caused $70 \%$ of the in- fections, among which $S$. aureus was the most common (41\%) followed by $S$. epidermidis (11\%). Our finding of gram-positive cocci as the main causative organisms of SSI is similar to the results of other series ${ }^{7,15,16)} . S$. aureus is a member of the normal flora of the body, frequently found in the nose, respiratory tract, and on the skin. Although $S$. aureus is not always pathogenic, it is a common cause of skin and soft tissue infections $^{26)}$. Nasal carriers of $S$. aureus are at increased risk for $S$. aureus $\mathrm{SSI}^{27)}$. A recent meta-analysis suggested that implementing a standard procedure including screening for nasal $S$. aureus, decolonizing carriers with mupirocin ointment, and chlorhexidine bathing before surgery reduced $S$. aureus SSIs $^{25)}$. Although decolonizing is not known to be effective in reducing SSI after neurosurgery, it may still be helpful in reducing SSI. Among gram-negative bacteria, Serratia marcescens was the most common (12\%). Serratia marcescens is an opportunistic pathogenic gram-negative bacillus that has emerged as a serious cause of nosocomial infections ${ }^{18)}$. Serratia marcescens is capable of thriving in diverse environments including water and soil, but most commonly in healthcare settings. In the last few decades, it has become more common in intensive care units (ICUs) as a cause of bacteremia, pneumonia, and urinary tract infections ${ }^{12,28,29)}$. Many patients who undergo neurosurgical procedures require ICU therapy, which may lead to infection by Serratia marcescens. To prevent it, thorough management of infections such as pneumonia and urinary tract infection is necessary.

Previously reported risk factors for SSI after neurosurgical procedures include cerebrospinal fluid (CSF) leak, multiple operations, operation time longer than 4 hours, higher ASA class, clean-contaminated or dirty wound, and surgeon $\operatorname{grade}^{2,3,15,17,22)}$. In our study, only preoperative hospital stay duration was found to be a risk factor for CRAN. In addition, an increase in over T-hour increased the risk of SSI, albeit the effect was not statistically significant. CSF leak, although identified as a risk factor in many studies, was not investigated because of the lack of data. The duration of preoperative hospital stay is lengthened by poor general condition, histories such as diabetes mellitus and hypertension, infection status such as pneumonia, urinary tract infection, and other complications in the case of trauma. These conditions can increase the risk of SSI. However, the poor general condition is not enough to explain the duration of preoperative hospital stay because other variables, such as ASA class and age, are also related to 
poor general condition. Future research will need additional analysis of the causes of longer preoperative periods. Long surgery is thought to increase the time of potential exposure to infection, often because of excessive bleeding.

This study has several limitations. First, although the total number of patients was high, the infection group size was small. Second, data of the presence of a CSF leak, ventricular drain, or foreign body, previously identified as risk factors, were limited and not analyzed. Additional data collection will be needed in future studies to analyze SSI in the neurosurgical field. Third, the diagnostic criteria for incisional infection included pus discharge, tenderness, local edema, redness, and fever at the time of bacterium isolation from the skin incision site or subcutaneous tissue culture. However, it is possible that the surgeon did not always diagnose infection when observing some of the above symptoms, reserving the diagnosis for cases with definitive evidence of infection such as pus at the skin incision site. Fourth, in accordance with the SSI criteria used, postoperative infections were defined as those occurring within 1 year of the operation when prosthetic material remained within the operation site. The SSI rate could be undervalued because our study analyzed preliminary data that was only 90 days in duration. In future studies, a 1-year monitoring period will be necessary.

In the meantime, a nationwide survey on SSI in Korea was conducted by internal medicine staff. However, there may be differences in the diagnosis of SSI judged by surgeons and physicians. This study was the first national survey of SSI under surgeon's diagnosis. Although there is a lack of finding risk factors for SSI due to the lack of analysis of various variables, we believe that it is also very meaningful to show reliable results on the SSI rate in the neurosurgical field. In future studies, when data is accumulated, and appropriate factors are analyzed, more valuable results can be obtained to help reduce SSI incidence.

\section{CONCLUSION}

SSIs can cause many serious problems in neurosurgery. Identifying SSI risk factors can help reduce mortality, morbidity, and improve patient care. An SSI surveillance system is important for the accurate analysis of SSI. The incidence of SSI after neurosurgical procedures assessed by a national sur- veillance system was $1.9 \%$. Preoperative hospital stay duration was an independent risk factor for SSI. Age and over T-hour tended to increase the risk of SSI.

\section{CONFLICTS OF INTEREST}

No potential conflict of interest relevant to this article was reported.

\section{INFORMED CONSENT}

Informed consent was obtained from all individual participants included in this study.

\section{References}

1. American Society of Anesthesiologists (ASA) : ASA physical status classification system. Available at : https://www.asahq.org/resources/clinical-information/asa-physical-status-classification-system

2. Blomstedt $\mathrm{GC}$ : Infections in neurosurgery: a retrospective study of 1143 patients and 1517 operations. Acta Neurochir (Wien) 78 : 81-90, 1985

3. Buang SS, Haspani MS : Risk factors for neurosurgical site infections after a neurosurgical procedure: a prospective observational study at Hospital Kuala Lumpur. Med J Malaysia 67 : 393-398, 2012

4. Centers for Disease Control and Prevention (CDC) : Surgical site infection (SSI) event. Available at : http://www.cdc.gov/nhsn/pdfs/ pscmanual/9pscssicurrent.pdf

5. Centers for Disease Control and Prevention (CDC) : CDC/NHSN Surveillance Definitions for Specific Types of Infections. Available at : https://www.cdc.gov/nhsn/pdfs/pscmanual/17pscnosinfdef_current. pdf

6. Centers for Disease Control and Prevention: Public health then and now: celebrating 50 years of MMWR at CDC. Available at: https://www.cdc.gov/mmwr/pdf/other/su6004.pdf

7. Chiang HY, Kamath AS, Pottinger JM, Greenlee JD, Howard III MA, Cavanaugh JE, et al. : Risk factors and outcomes associated with surgical site infections after craniotomy or craniectomy. J Neurosurg 120 : 509-521, 2014

8. Dixon RE, Centers for Disease Control and Prevention (CDC) : Control of health-care-associated infections, 1961-2011. MMWR Suppl 60 : 58 63, 2011

9. Emori TG, Gaynes RP : An overview of nosocomial infections, including the role of the microbiology laboratory. Clin Microbiol Rev 6 : 428442, 1993 
10. Gastmeier P, Kampf G, Wischnewski N, Hauer T, Schulgen G, Schumacher $\mathrm{M}$, et al. : Prevalence of nosocomial infections in representative German hospitals. J Hosp Infect 38 : 37-49, 1998

11. Gaynes RP, Culver DH, Horan TC, Edwards JR, Richards C, Tolson JS, et al. : Surgical site infection (SSI) rates in the United States, 1992-1998: the National Nosocomial Infections Surveillance System basic SSI risk index. Clin Infect Dis 33 (Suppl 2) : S69-S77, 2001

12. Hadid H, Usman M, Thapa S : Severe osteomyelitis and septic arthritis due to serratia marcescens in an Immunocompetent Patient. Case Rep Infect Dis $2015: 347652,2015$

13. Haley RW, Culver DH, White JW, Morgan WM, Emori TG, Munn VP, et al. : The efficacy of infection surveillance and control programs in preventing nosocomial infections in US hospitals. Am J Epidemiol 121 : 182-205, 1985

14. Hall WA, Truwit $\mathrm{CL}$ : The surgical management of infections involving the cerebrum. Neurosurgery 62 (Suppl 2) : SHC519-SHC530; discussion 530-531, 2008

15. Korinek $A M$ : Risk factors for neurosurgical site infections after craniotomy: a prospective multicenter study of 2944 patients. The French Study Group of Neurosurgical Infections, the SEHP, and the C-CLIN Paris-Nord. Service Epidémiologie Hygiène et Prévention. Neurosurgery 41 : 1073-1079; discussion 1079-1081, 1997

16. Korinek A, Golmard J, Elcheick A, Bismuth R, Van Effenterre R, Coriat P, et al. : Risk factors for neurosurgical site infections after craniotomy: a critical reappraisal of antibiotic prophylaxis on 4578 patients. Br J Neurosurg $19: 155-162,2005$

17. Kourbeti IS, Jacobs AV, Koslow M, Karabetsos D, Holzman RS : Risk factors associated with postcraniotomy meningitis. Neurosurgery 60 : 317-325; discussion 325-326, 2007

18. Lew DP, Waldvogel FA : Osteomyelitis. The Lancet 364 : 369-379, 2004

19. Mangram AJ, Horan TC, Pearson ML, Silver LC, Jarvis WR, The Hospital Infection Control Practices Advisory Committee : Guideline for preven- tion of surgical site infection, 1999. Am J Infect Control 27 : 97-132; quiz 133-134; discussion 96, 1999

20. McClelland III S, Hall WA : Postoperative central nervous system infection: incidence and associated factors in 2111 neurosurgical procedures. Clin Infect Dis 45 : 55-59, 2007

21. McLaws ML, Gold J, King K, Irwig LM, Berry G : The prevalence of nosocomial and community-acquired infections in Australian hospitals. Med J Aust 149 : 582-590, 1988

22. Narotam PK, van Dellen JR, du Trevou MD, Gouws E : Operative sepsis in neurosurgery: a method of classifying surgical cases. Neurosurgery 34 : 409-415; discussion 415-416, 1994

23. Patir R, Mahapatra AK, Banerji AK : Risk factors in postoperative neurosurgical infection. A prospective study. Acta Neurochir (Wien) 119 : 80-84, 1992

24. Plowman $R$ : The socioeconomic burden of hospital acquired infection. Euro Surveill $5:$ 49-50, 2000

25. Schweizer M, Perencevich E, McDanel J, Carson J, Formanek M, Hafner J, et al. : Effectiveness of a bundled intervention of decolonization and prophylaxis to decrease Gram positive surgical site infections after cardiac or orthopedic surgery: systematic review and meta-analysis. BMJ $346: \mathfrak{f} 2743,2013$

26. Tong SY, Davis JS, Eichenberger E, Holland TL, Fowler VG Jr : Staphylococcus aureus infections: epidemiology, pathophysiology, clinical manifestations, and management. Clin Microbiol Rev 28 : 603-661, 2015

27. von Eiff C, Becker K, Machka K, Stammer H, Peters $G$ : Nasal carriage as a source of Staphylococcus aureus bacteremia. N Engl J Med 344 : 11 16, 2001

28. Wilhelmi I, de Quirós JB, Romero-Vivas J, Duarte J, Rojo E, Bouza E : Epidemic outbreak of Serratia marcescens infection in a cardiac surgery unit. J Clin Microbiol 25 : 1298-1300, 1987

29. Yu VL : Serratia marcescens: historical perspective and clinical review. $\mathbf{N}$ Engl J Med 300 : 887-893, 1979 